\title{
Hepatomegaly, weight loss and general malaise - the first manifestations of primary systemic amyloidosis
}

\author{
Halina Cichoż-Lach ${ }^{1}$, Beata Prozorow-Król1, Jarosław Swatek², Barbara Skrzydło-Radomańska', Leszek Buk ${ }^{3}$, \\ Małgorzata Zdunek ${ }^{2}$, Agnieszka Kowalik ${ }^{1}$, Maria Słomka ${ }^{1}$ \\ ${ }^{1}$ Department of Gastroenterology, Medical University of Lublin, Poland \\ ${ }^{2}$ Department of Clinical Pathomorphology, Medical University of Lublin, Poland \\ ${ }^{3} 1^{\text {st }}$ Department of Radiology, Medical University of Lublin, Poland
}

Prz Gastroenterol 2014; 9 (1): 57-61

DOI: $10.5114 /$ pg.2014.40853

Key words: amyloidosis, histological diagnosis, hepatomegaly.

Address for correspondence: Halina Cichoż-Lach MD, PhD, Department of Gastroenterology, Medical University of Lublin, 8 Jaczewskiego St, 20-954 Lublin, Poland, phone: +48 8174307 36, fax: +48 8147961 35, e-mail: lach.halina@wp.pl

\begin{abstract}
Amyloidosis is characterised by the accumulation of poorly soluble fibrous proteins in the extracellular space of various bodily organs. Light chain amyloidosis (AL) is recognised as the most common form of systemic amyloidosis. Light chains are deposited in the majority of bodily organs, and accumulation of them in the liver produces hepatomegaly. We report a case of AL-systemic amyloidosis with liver involvement in a 71-year-old woman. Hepatomegaly, weight loss and general malaise were the first manifestations of the disease. Liver biopsy found amyloid deposits along the sinusoids as well as in the space of Disse, inside the vascular wall and in connective tissue of the portal tracts, which showed a positive reaction in Congo Red stain. Further diagnosis showed the presence of systemic amyloidosis. The patient was put on cyclophosphamide and steroid therapy.
\end{abstract}

\section{Introduction}

Amyloidosis is characterised by accumulation of poorly soluble fibrous proteins known as amyloids in the extracellular spaces of various bodily organs. Depending on the kind of deposited proteins, over ten types of amyloidosis are distinguished, including systemic and organ amyloidosis. Light chain amyloidosis $(\mathrm{AL})$, also called primary amyloidosis, is recognised as the most common form of systemic amyloidosis. Light chains are deposited in the majority of bodily organs, most commonly in the kidneys, the heart and the peripheral nervous system [1]. Accumulation of amyloids in the liver produces hepatomegaly in $33-92 \%$ of patients, as well as moderate jaundice and moderate to severe cholestasis, but hardly ever results in organ insufficiency or portal hypertension that may be accompanied by sub-capsular haematomas and spontaneous rupture of the liver $[2,3]$.This type of amyloidosis may also be present in multiple myeloma.

Secondary amyloidosis involves deposition of SAA protein (serum amyloid $A$ ), and it can occur in the course of a chronic inflammatory or non-inflammatory disease, lymphomas and some genetic diseases.

The prognosis in amyloidosis is poor; it has been estimated that the mean survival rate for patients with amyloidosis is 10-14 months following diagnosis [4]. There are no estimates specifying the prevalence of amyloidosis in Poland. In the USA it is diagnosed in 5.1-12.8 patients/ 1 million people a year, and $90 \%$ of the patients are over 50 years old [5] .

The aim of this study is the presentation of primary systemic amyloidosis with liver involvement. Hepatomegaly was the first manifestation of the disease.

\section{Case report}

A 71-year-old woman was admitted to the Department of Gastroenterology at the Medical University of Lublin because of general malaise and $10 \mathrm{~kg}$ weight loss within 1 month.

The patient did not report any other ailments. She had been treated for arterial hypertension for the previous 10 years (II degree according to WHO) and was taking perindopril - $4 \mathrm{mg} /$ daily and nitrendipine $-20 \mathrm{mg}$ / 

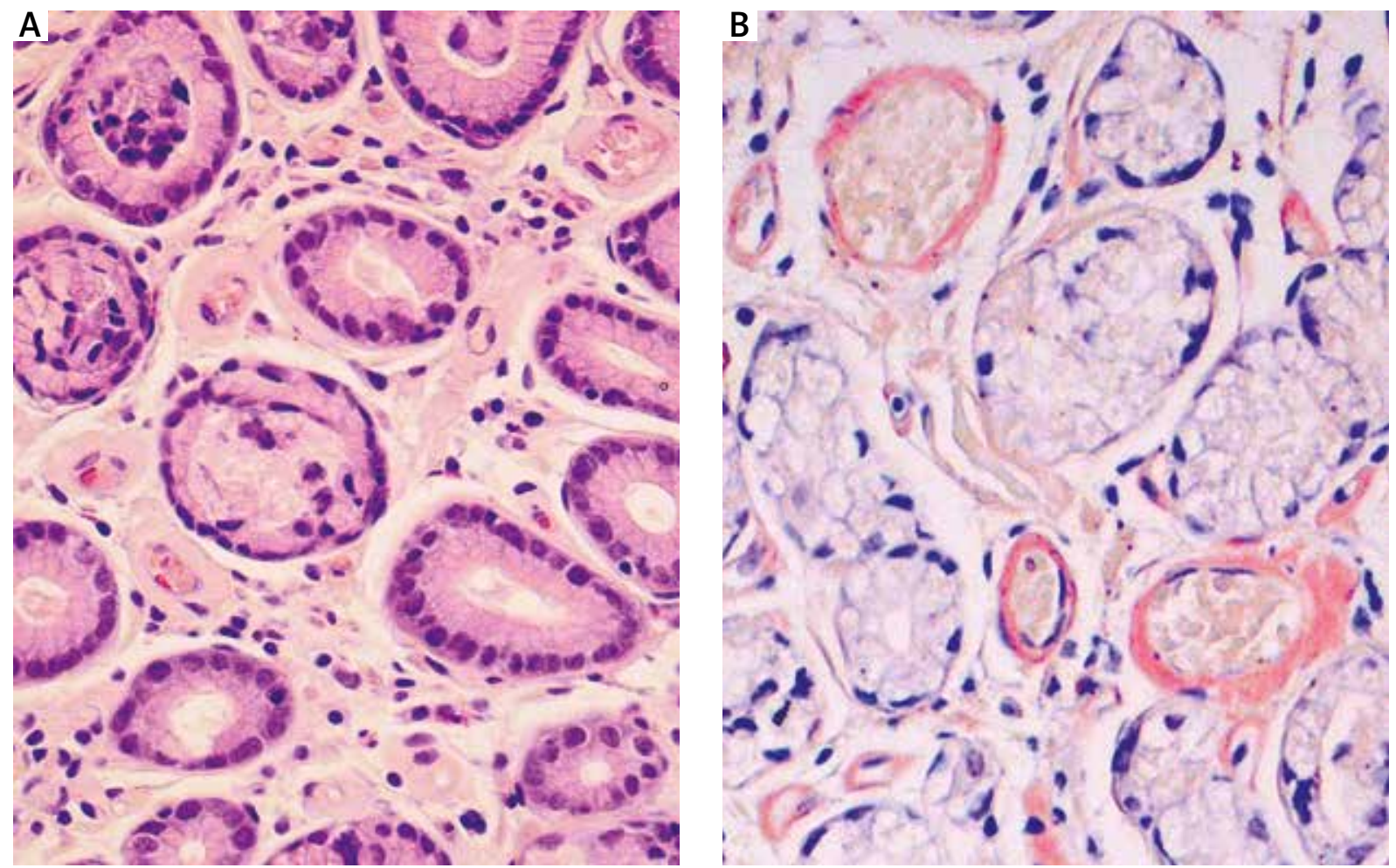

Figure 1. Annular deposits of amyloid around capillaries of gastric mucosa (200x; A - H + E; B - Congo Red)

daily, on a regular basis. A gynaecological interview was of no clinical relevance. The patient's mother died of liver cancer at the age of 79. The patient was a non-smoker and she abstained from alcohol.

On admission the physical examination revealed a vesicular murmur over the lung field, normal heart sounds, pulse $72 \mathrm{bpm}$ and arterial blood pressure 140/ $80 \mathrm{~mm} \mathrm{Hg}$. The liver was enlarged, palpable about $15 \mathrm{~cm}$ beneath the subcostal arch in the right midclavicular line and $8 \mathrm{~cm}$ in the body midline, and it was hard with a smooth, sharp edge. The spleen was not palpable.

Laboratory tests revealed: normal values of blood morphology, coagulation system, bilirubin, lipidogram, glucose, sodium, potassium, iron, ferritin and amylase. Increased activity of alanine aminotransferase (ALT) $43 \mathrm{U} / \mathrm{l}$ (norm up to $31 \mathrm{U} / \mathrm{l}$ ) and asparagine aminotransferase (AST) - $75 \mathrm{U} / \mathrm{I}$ (norm up to $34 \mathrm{U} / \mathrm{l}$ ) were observed, as well as the activities of: alkaline phosphatase (ALP) - $266 \mathrm{U} / \mathrm{l}$ (norm 36-111 U/l), GGTP - $219 \mathrm{U} / \mathrm{l}$ (norm 11-35 U/l), urea level- $131.1 \mathrm{mg} / \mathrm{dl}$ (norm 12.8-43 mg/ $\mathrm{dl}$ ) and creatinine $-2.7 \mathrm{mg} / \mathrm{dl}$ (norm $0.5-1.1 \mathrm{mg} / \mathrm{dl}$ ). The level of $\alpha$-fetoprotein was normal. The total protein level was $5.5 \mathrm{~g} / \mathrm{dl}$ (norm 6.4-8.3 g/dl) with a decreased albumin level - $2.62 \mathrm{~g} / \mathrm{dl}$ (norm 3.2-4.8 g/dl), increased concentration of $\alpha 1$ globulin $-0.35 \mathrm{~g} / \mathrm{dl}$ (norm $0.08-0.23$ $\mathrm{g} / \mathrm{dl}$ ) and $\beta 2$ globulin $-0.58 \mathrm{~g} / \mathrm{dl}$ (norm $0.18-0.5 \mathrm{~g} / \mathrm{dl}$ ).
On screening examination, antinuclear autoantibodies were positive (titre $1: 320$ ), and no other autoantibodies characteristic for autoimmune hepatitis were detected. Infection with hepatotropic viruses (HBV, HCV, CMV, EBV) was ruled out.

Panendoscopy showed a single, first-degree oesophageal varix, erythemas in the prepyloric part of the stomach and contact bleeding of the mucus in this area. Biopsy specimens from the subcardial area, the body of the stomach and the prepyloric area, on Congo Red staining with the use of a polarised light microscope as well as in fluorescent staining with Thioflavin T, presented deposits of amyloid along the border of the glandular basement membranes and around capillaries of gastric mucosa (Figure 1).

An abdominal ultrasound found hepatomegaly of intensified echogenicity together with pronounced intrahepatic bile ducts. In the gall bladder a polyp of approximately $4 \mathrm{~mm}$ diameter was detected. Apart from that, all the abdominal and pelvic organs remained unchanged. An abdominal Doppler ultrasound revealed normal hepatic veins with a monophasic blood flow out of the liver. The portal vein was $10 \mathrm{~mm}$ wide and its intrahepatic branches were patent; the direction of blood flow was correct with $\mathrm{V}_{\max }$ in inspiration $-22 \mathrm{~cm} / \mathrm{s}$. The splenic vein was patent and was not widened. 


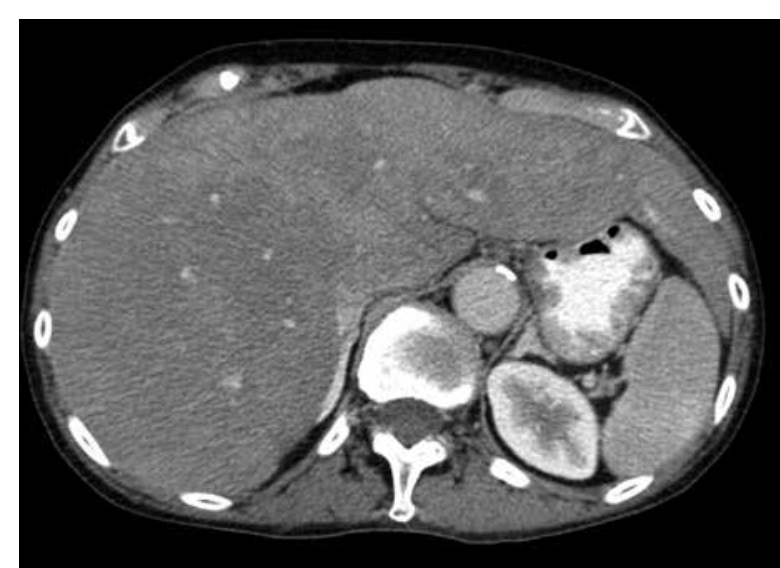

Figure 2. The CT scan - reduced hepatic perfusion in enlarged liver after injection of a contrast medium bolus

An X-ray of the lungs revealed a normal view of the lung parenchyma. However, a marked enlargement of the heart silhouette in the range of the left ventricle as well as the presence of atheromatous plaque in the aortic arch were detected.

A computed tomography (CT) scan of the chest, abdomen and small pelvis showed a correct lung parenchyma as well as numerous nodulous changes in the thyroid and a substantially enlarged liver with reduced hepatic perfusion after an injection of contrast medium (Figure 2). All the remaining abdominal organs presented no pathological changes.

Because of considerable hepatomegaly it was decided to perform a liver biopsy. Histopathological examination results revealed a significantly impaired structure of the hepatic lobules due to atrophy and degeneration of the majority of hepatocytes. Deposits of amyloid were found along the sinusoids, in the space of Disse and inside the vascular wall and connective tissue of the portal tracts (Figure 3). Distorted portal tracts, intensely infiltrated with lymphocytes and plasmocytes, as well as single neutrophil granulocytes, were also noted. The vascular wall was thickened. In histopathological staining, the analysed deposits showed a positive reaction to Congo Red staining and a negative reaction after diastase digestion, whereas fluorescent staining with Thioflavin $T$ revealed the presence of amyloid deposits, which was suggestive of amyloidosis. The histochemical reaction to $\mathrm{HBsAg}$ was negative.

Sigmoidoscopy did not show changes in the macroscopic picture of the large intestine. A histopathological examination of the specimens obtained from the rectum proved the presence of amyloid deposits in the walls of tiny vessels in the mucous membrane area in the Congo

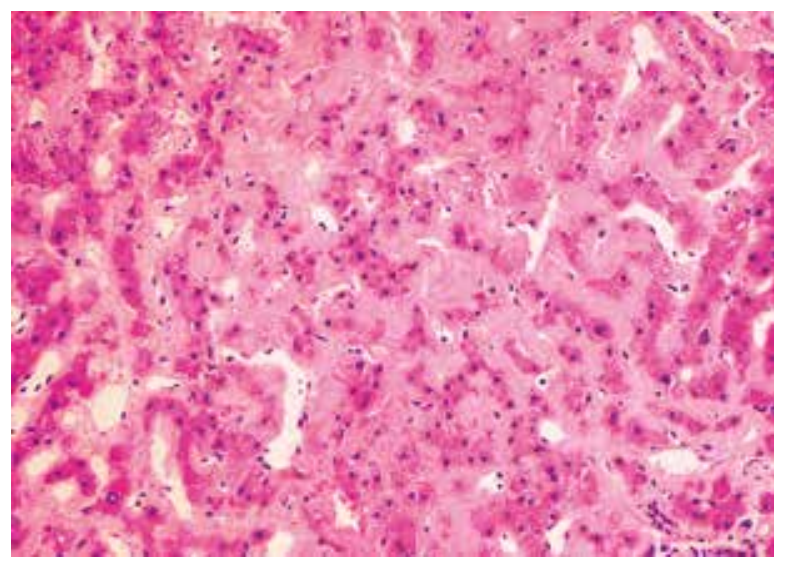

Figure 3. Extensive deposits of amyloid between sinusoids and hepatocytes (in the spaces of Disse) with atrophy of hepatocytes $(H+E, 100 x)$

Red stain with the use of a polarised microscope as well as in fluorescent staining with Thioflavin T.

Further laboratory blood tests detected increased values of $\beta 2$-microglobulin - $15.5 \mathrm{mg} / \mathrm{l}$ (norm $0.7-1.8 \mathrm{mg} / \mathrm{l}$ ), high values of the titre of free $\kappa$ chains $-473 \mathrm{mg} / \mathrm{l}$ (norm up to $19.4 \mathrm{mg} / \mathrm{l}$ ), a marked protein increase in 24-hour urine collection - $1054.2 \mathrm{mg}$ (norm 0.0-150 mg/day) and decreased creatinine excretion in $24 \mathrm{~h}$ urine collection - 252.4 mg (norm 600-1800 mg/day). No monoclonal proteins were found in the blood serum with the use of immunofixation electrophoresis technique (IFE), and the Bence-Jones protein was not detected in the urine, either. A heart echocardiography performed in M-mode, 2D showed extensive hypertrophy of the left ventricle and its impaired relaxation, relaxative (diastolic) dysfunction of the left ventricle and ejection fraction $\mathrm{EF}-60 \%$. The electrocardiogram (ECG) presented negative $T$ waves in $\mathrm{aVL}$ and $\mathrm{V} 5-\mathrm{V} 6$, hypertrophic features, and left ventricle overload. Moreover, a trepanobiopsy of the bone marrow was performed and it revealed the presence of protein deposits of an amyloid nature, with no other clinically significant pathologies. On routine examination, point mutation JAK-2 was not detected, ruling out true polycythaemia.

Throughout the hospitalisation period the patient's general condition was good, her only complaint being persistent weakness. Following nephrological and haematological consultation, primary systemic amyloidosis was diagnosed. Further monitoring of the renal parameters was recommended and steroid therapy with dexamethasone $-20 \mathrm{mg}$ orally on the $1^{\text {st }}, 4^{\text {th }}, 8^{\text {th }}$ and $15^{\text {th }}$ days of treatment was launched. Subsequently, the patient was put on cyclophosphamide - $500 \mathrm{mg} /$ daily while administration of dexamethasone was still continued. 


\section{Discussion}

Patients with primary amyloidosis generally present light chain deposits in all organs, yet amyloidosis hardly ever affects the central nervous system. Most frequently the disease affects the kidneys, where the deposits build up in the renal glomerulus and interstitial tissue. As a result of this proteinuria, nephritic syndrome and renal failure develop, which requires application of dialysis therapy in up to approximately $20 \%$ of patients [6] In the described case the biochemical features of renal dysfunction were present but they were not of such severity as to require kidney substitute therapy. However, the hepatic changes dominant in the clinical picture were much more intensified. A considerably enlarged liver was the first symptom, and it triggered further investigations which, eventually, resulted in diagnosing amyloidosis.

Hepatomegaly is quite common in the course of amyloidosis, but it is quite infrequently accompanied by organ dysfunction [7]. Amyloid deposits are present along the sinusoids, in the space of Disse, in the vascular wall and in connective tissue of the portal tracts, which results in dysfunction of the hepatic lobules as well as degeneration of the majority of hepatocytes. The presented patient demonstrated a characteristic histological picture. The liver function parameters showed poor improvements; the features of cholestasis, however, which manifested themselves as increased activity of ALP and GGTP, according to the data found in the literature, appear to be very characteristic of this condition. Many authors emphasise the moderate increase in ALP activity as well as substantial organ enlargement that often accompanies it as typical in the course of this disease [8]. In the presented patient the hepatomegaly was very severe and the liver biopsy confirmed amyloidosis. The presence of an oesophageal varix was suggestive of the onset of portal hypertension despite the correct parameters of vascular flow presented in the Doppler ultrasound. Identification of amyloid deposits in the liver extended the range of diagnostic investigations, which resulted in detecting amyloid deposits in other organs and allowed for the diagnosis of systemic amylosis.

Patients suffering from amyloidosis often present digestive system complaints that may manifest themselves by disturbed defecation rhythm, lack of appetite and latent haemorrhages [9]. They are all results of direct impairment of the mucous membrane and its vessels, as well as of the changes of neuropathic nature that are associated with amyloid deposition.

The changes revealed by the ECG and ECHO of the heart were very typical of light chain amyloidosis. Cardiac amyloidosis manifests itself by a circulatory failure, whereas in the ultrasound images a substantial hypertrophy of the left ventricle and its disturbed relaxation were dominant.

The treatment of amyloidosis involves treating the basic disease (monoclonal gammapathy of multiple myeloma) with the use of cytostatics (melphalan), steroids and autologous bone marrow transplant $[10,11]$. The inefficient organs require symptomatic treatment and the general prognosis is bad [12-14]. With involvement of the kidneys, the mean survival time is $12-18$ months, and in the case of heart involvement it is 6 months. The survival period for patients who present laboratory features of liver dysfunction is 14 months [6]. Even though systemic amyloidosis is an infrequent disease, it is a multi-organ pathology resulting in substantially compromised functioning of the whole organism. If left untreated it leads rapidly to progressive renal failure, circulatory failure and liver impairment and it is often fatal.

It is mandatory that in the presence of hepatomegaly accompanied by liver dysfunction, even of a mild nature, not only common liver pathologies, such as hepatotropic virus infections or conditions of autoimmunological or toxic origin, should be taken into consideration, but also less typical ones, for instance light chain amyloidosis. Diagnosing hepatic amyloidosis requires further investigations to confirm systemic amyloidosis so that optimal treatment, both causative and symptomatic, can be implemented. It must be remembered that amyloidosis causes multi-organ insufficiency and therefore intensive interdisciplinary cooperation is indispensable in the management of this pathology.

\section{References}

1. Lachmann HJ, Goodman HJ, Gilbertson JA, et al. Natural history and outcome in systemic AA amyloidosis. N Engl J Med 2007; 356: 2361-71.

2. Bujanda L, Beguiristain A, Alberdi F, et al. Spontaneous rupture of the liver in amyloidosis. Am J Gastroenterol 1997; 92: 1385-6.

3. Tam M, Seldin DC, Forbes BM, et al. Spontaneous rupture of the liver in a patient with systemic AL amyloidosis undergoing treatment with high-dose melphalan and autologous stem cell transplantation: a case report with literature review. Amyloid 2009; 16: 103-7.

4. Skinner M, Anderson J, Simms R, et al. Treatment of 100 patients with primary amyloidosis: a randomized trial of melphalan, prednisone, and colchicine versus colchicine only. Am J Med 1996; 100: 290-8.

5. Roszyk E, Chmielewski M, Linke K, et al. Severe intrahepatic cholestasis in a patient with AL amyloidosis [Polish]. Prz Gastroenterol 2010; 5: 110-5.

6. Pozzi C, Locatelli F. Kidney and liver involvement in monoclonal light chain disorders. Semin Nephrol 2002; 22: 319-30. 
7. Joshi D, Belgaumkar A, Ratnayake V, et al. A case of hepatomegaly. Postgrad Med J 2007; 83: e1-2.

8. Altraif I, Handoo FA, Alsaad KO, et al. Fatal subacute hepatic failure in a patient with AA-type amyloidosis: case report. Patholog Res Int 2010; 2010: 648089.

9. Ziółkowski BA, Pacholec A, Kudlicka M, et al. Prevalence of abdominal symptoms in the Polish population. Prz Gastroenterol 2012; 7: 20-5.

10. Sanchorawala V, Seldin DC. An overview of high-dose melphalan and stem cell transplantation in the treatment of $\mathrm{AL}$ amyloidosis. Amyloid 2007; 14: 261-9.

11. Sanchorawala V, Skinner M, Quillen K, et al. Long-term outcome of patients with $\mathrm{AL}$ amyloidosis treated with high-dose melphalan and stem-cell transplantation. Blood 2007; 110: 3561-3.

12. Sanchorawala V, Wright DG, Seldin DC, et al. An overview of the use of high-dose melphalan with autologous stem cell transplantation for the treatment of $\mathrm{AL}$ amyloidosis. Bone Marrow Transplant 2001; 28: 637-42.

13. Skinner M, Sanchorawala V, Seldin DC, et al. High-dose melphalan and autologous stem-cell transplantation in patients with AL amyloidosis: an 8-year study. Ann Intern Med 2004; 140: 85-93.

14. Girnius S, Seldin DC, Skinner M, et al. Hepatic response after high-dose melphalan and stem cell transplantation in patients with $\mathrm{AL}$ amyloidosis associated liver disease. Haematologica 2009; 94: 1029-32.

Received: $\quad 2.11 .2011$

Accepted: $\quad 29.03 .2012$ 